\title{
Depression Risk and Outcomes Among ASCVD Patients
}

\author{
Ron D. Hays, PhD \\ Division of General Internal Medicine \& Health Services Research, UCLA Department of Medicine, Los Angeles, CA, USA.
}

$\mathrm{J}$ Gen Intern Med 35(8):2478

DOI: $10.1007 / \mathrm{s} 11606-020-05789-\mathrm{Z}$

(c) Society of General Internal Medicine 2020

$\mathrm{O}$ kunrintemi et al. ${ }^{1}$ found that those who scored between 4 and 6 ("high risk" for depression) versus between 0 and 3 ("low risk" for depression) on the 2-item Patient Health Questionnaire depression had worse SF-12 PCS and MCS scores and worse reported self-rated general health (In general, would you say your health is: excellent, very good, good, fair, poor). In addition, those with a clinical diagnosis of depression (ICD-9-M Code of 311) had worse SF-12 PCS and MCS scores and self-rated health than those without depression. The finding that depressive symptoms and depression are associated with SF-12 MCS scores is circular because the SF-12 assesses mental health. While the standard scoring of the SF-12 forces the PCS and MCS to be uncorrelated, depression is correlated with physical health. ${ }^{2}$ In addition, the self-rated general health item is one of the SF-12 questions.

Moreover, the authors incorrectly state that the SF-12 PCS and MCS scores range from " 0 to (worst health status possible) to 100 (best health status possible)" (p. 2429). The PCS and MCS are scored on a T-score metric with a mean of 50 and standard deviation of 10 in the US general population.

The authors refer to the excellent, very good, good, fair, and poor response scale as a "Likert scale."” Technically, a Likert scale uses an agree-disagree response scale. Not all polytomous response scales are Likert scales. ${ }^{3}$

The authors call the CAHPS overall rating of healthcare item a measure of patient satisfaction. No articles published by the CAHPS instrument developers are cited and there is no reference to the CAHPS website ${ }^{4}$ where it is noted that the "terms patient satisfaction and patient experience are often used interchangeably, but they are not the same thing."

Corresponding Author: Ron D. Hays, PhD; Division of General Internal Medicine \& Health Services Research UCLA Department of Medicine, 1100 Glendon Avenue, Suite 850, Los Angeles, CA 90024, USA (e-mail: drhays@ucla.edu).

\section{REFERENCES}

1. Okunrintemi V, Valero-Elizondo Micos ED, Salami JA, et al. Association of depression risk with patient experience, healthcare experience, and health resource utilization among adults with atherosclerotic cardiovascular disease. J Gen Intern Med. 2019;34:2427-34.

2. Farivar SS, Cunningham WE, Hays RD. Correlated physical and mental health summary scores for the SF-36 and SF-12 health survey, V. 1. Health Qual Life Outcomes. 2007;5:54.

3. Uebersax JS. Likert Scales: Dispelling the Confusion. Available at: http:// www.john-uebersax.com/stat/likert.htm. Accessed November 20, 2019.

4. Agency for Healthcare Research and Quality. What is Patient Experience? Available at: https://www.ahrq.gov/cahps/about-cahps/patient-experience/index.html. Accessed November 20, 2019.

Publisher's Note: Springer Nature remains neutral with regard to jurisdictional claims in published maps and institutional affiliations. 